\title{
A literature review on mobile devices touch screen inputs and its techniques evaluation
}

\begin{abstract}
Due to the proliferation of touch screen devices, both technical and non-technical users are increasingly using these devices including the older adults. Interaction with input devices while on the go is increasing and has become a common habit of people nowadays. In fact, at times it might also be a need for different people of different age groups. Common sense leads to believe that performing tasks that require high mental workload such as text input in walking state, can prove fatal as the users visual attention gets split between the environment and the device. Situational impairment can happen because of environmental, attentional and physical factors at various times while the user is operating the device. Previous studies have measured the negative impact of mobility on text input performance of young adults, expert users on touch screen devices of different sizes. In this paper, we present the research studies done on text input in mobility conditions and methods used for input method evaluation.
\end{abstract}

Keyword: Mobility; Older adults; Static; Text input; Touch screen device 